KA-TP-24-1999

August 13, 2018

\title{
Hopf instantons and the Liouville equation in target space
}

\author{
C. Adam* \\ Institut für Theoretische Physik, Universität Karlsruhe \\ B. Muratori**, C. Nash*** \\ Department of Mathematical Physics, National University of Ireland, Maynooth
}

\begin{abstract}
We generalise recent results on Hopf instantons in a Chern-Simons \& Fermion theory in a fixed background magnetic field. We find that these instanton solutions have to obey the Liouville equation in target space. As a consequence, these solutions are given by a class of Hopf maps that consist of the composition of the standard Hopf map with an arbitrary rational map.
\end{abstract}

*)email address: adam@maths.tcd.ie, adam@pap.univie.ac.at

**)email address: bmurator@fermi1.thphys.may.ie

***)email address: cnash@stokes2.thphys.may.ie 


\section{Introduction}

In this letter, we shall generalise some recent results [1] on fully three-dimensional solutions of an Abelian Chern-Simons and Fermion system in the presence of a fixed background magnetic field. These solutions are related to Hopf maps and are, therefore, labelled by the Hopf index. Hopf maps are just maps $S^{3} \rightarrow S^{2}$. These maps fall into different homotopy classes that are labelled by the integers (the Hopf index, see below for details). Field theories where static solutions with nontrivial Hopf index (Hopf solitons) occur have already received considerable interest recently (see e.g., [2]-8]).

Chern-Simons theories have been widely studied ever since their introduction [9]. Specifically, when an Abelian Chern-Simons term in three dimensions is coupled to matter, the magnetic field is forced to be proportional to the electric current due to the equations of motion [10]-[14]. Further, in these models there exist soliton-like, static (i.e., two-dimensional) solutions that are related to some topological invariants (e.g., maps $S^{2} \rightarrow S^{2}$ [10] 14. Usually, these solitons behave like vortices, and, because of their topological nature, they exhibit magnetic flux quantization. Therefore these solutions are physically relevant in situations where the phenomenon of magnetic flux quantization occurs and where matter is confined to a plane, the most prominent example being the quantum Hall effect [15, 16].

At this point the question arises whether there exist fully three-dimensional solutions for such Chern-Simons \& matter systems, and whether these solutions may be characterized by some topological invariants, as well.

In a recent paper [1] we have demonstrated that, if the presence of a fixed, prescribed background magnetic field is assumed, then there indeed exist solutions to the ChernSimons \& Fermion system defined below. These solutions are related to the specific Hopf maps

$$
S^{3} \stackrel{\chi}{\rightarrow} S^{2} \stackrel{R_{n}}{\rightarrow} S^{2}
$$

where $\chi$ is the standard Hopf map with Hopf index 1 and

$$
R_{n}: z \rightarrow z^{n}, \quad z \in \mathbf{C}, \quad n \in \mathbf{Z}
$$

is a special class of rational maps $\mathbf{C} \rightarrow \mathbf{C}$ that express maps $S^{2} \rightarrow S^{2}$ in stereographic coordinates (see below for details). The Hopf maps (1) have Hopf index $N=n^{2}$, and $n$ is the winding number of the corresponding map $S^{2} \rightarrow S^{2},(2)$.

In this letter we shall show that the special class (2) of rational maps in (1) may actually be generalised to arbitrary rational maps $R(z)$. These general rational maps will emerge as solutions of the Liouville equation on the target $S^{2}$ in (1) that the Hopf instantons will be proven to obey.

The paper is organised as follows. In Section 2 we briefly review some features of maps $S^{2} \rightarrow S^{2}$ and of Hopf maps $S^{3} \rightarrow S^{2}$. In Section 3, we define our Chern-Simons and Fermion system. We show that its solutions in the presence of a fixed prescribed background magnetic field are solutions to the Liouville equation in target space. Consequently, these solutions are given by general rational maps in target space. We discuss our results in the final section. 


\section{Maps $S^{2} \rightarrow S^{2}$ and Hopf maps $S^{3} \rightarrow S^{2}$}

Maps $S^{2} \rightarrow S^{2}$ are characterised by their winding number $w$. One way of describing them is by interpreting both $S^{2}$ as Riemann spheres and by introducing stereographic coordinates $z \in \mathbf{C}$ on both of them. A specific class of such maps $S^{2} \rightarrow S^{2}$ may then be described by rational maps

$$
R: z \rightarrow R(z)=\frac{P(z)}{Q(z)}
$$

where $P(z)$ and $Q(z)$ are polynomials, and $z$ and $R(z)$ are interpreted as stereographic coordinates on the domain and target $S^{2}$, respectively. The winding number $w$ of this map is given by the degree of the map,

$$
w=\operatorname{deg}(R)=\max (p, q)
$$

where $p$ and $q$ are the degrees of the polynomials $P(z)$ and $Q(z)$. Another possibility of computing the same winding number involves the pullback under $R(z)$ of the standard area two-form $\Omega$ on $S^{2}$ (in stereographic coordinates),

$$
\Omega=\frac{2}{i} \frac{d \bar{z} d z}{(1+z \bar{z})^{2}}, \quad \int \Omega=4 \pi .
$$

The pullback is (' means derivative w.r.t. the argument)

$$
R^{*} \Omega=\frac{2}{i} \frac{\left|R^{\prime}(z)\right|^{2}}{(1+R \bar{R})^{2}} d \bar{z} d z=: \frac{2}{i} \rho_{R}(z, \bar{z}) d \bar{z} d z
$$

and obeys

$$
\int R^{*} \Omega=4 \pi w
$$

where $w$ is again the winding number (4). For later convenience we want to point out that the function $\rho_{R}(z, \bar{z})$ defined in (6) obeys the Liouville equation for all rational maps $R(z)$ (in fact for all holomorphic functions $f(z)$; however, we will be forced to restrict to rational maps later on by regularity requirements),

$$
\partial_{z} \partial_{\bar{z}} \ln \rho_{R}(z, \bar{z})=-2 \rho_{R}(z, \bar{z})
$$

where

$$
\partial_{z} \partial_{\bar{z}}=\frac{1}{4}\left(\partial_{x}^{2}+\partial_{y}^{2}\right)=\frac{1}{4} \Delta
$$

( $\Delta$ is the two-dimensional Laplacian) and we expressed $z$ by its real and imaginary part,

$$
z=x+i y, \quad \partial_{z}=\frac{1}{2}\left(\partial_{x}-i \partial_{y}\right)
$$

If we separate the function $\rho_{z}(z, \bar{z})=(1+z \bar{z})^{-2}$ that corresponds to the identity map $z \rightarrow z$ from $\rho_{R}(z, \bar{z})$,

$$
\rho_{R}=: \rho_{z} \widetilde{\rho}_{R}
$$


then $\widetilde{\rho}_{R}$ obeys the equation

$$
\partial_{z} \partial_{\bar{z}} \ln \widetilde{\rho}_{R}=-2 \frac{\widetilde{\rho}_{R}-1}{(1+z \bar{z})^{2}}
$$

which we shall need later on.

Hopf maps are maps $S^{3} \rightarrow S^{2}$. They may be expressed, e.g., by maps $\chi: \mathbf{R}^{3} \rightarrow \mathbf{C}$ provided that the complex function $\chi$ obeys $\lim _{|\vec{x}| \rightarrow \infty} \chi(\vec{x})=\chi_{0}=$ const, where $\vec{x}=$ $\left(x_{1}, x_{2}, x_{3}\right)^{\mathrm{T}}$. The pre-images in $\mathbf{R}^{3}$ of points of the target $S^{2}$ (i.e., the pre-images of points $\chi=$ const) are closed curves in $\mathbf{R}^{3}$ (circles in the related domain $S^{3}$ ). Any two different circles are linked $N$ times, where $N$ is the Hopf index of the given Hopf map $\chi$. Further, a magnetic field $\vec{B}$ (the Hopf curvature) is related to the Hopf map $\chi$ via

$$
\vec{B}=\frac{2}{i} \frac{(\vec{\partial} \bar{\chi}) \times(\vec{\partial} \chi)}{(1+\bar{\chi} \chi)^{2}}=4 \frac{S(\vec{\partial} S) \times \vec{\partial} \sigma}{\left(1+S^{2}\right)^{2}}
$$

where $\chi=S e^{i \sigma}$ is expressed in terms of its modulus $S$ and phase $\sigma$ at the r.h.s. of (13).

Mathematically, the curvature $F=\frac{1}{2} F_{i j} d x_{i} d x_{j}, F_{i j}=\epsilon_{i j k} B_{k}$, is the pullback under the Hopf map, $F=\chi^{*} \Omega$, of the standard area two-form $\Omega$, (5), on the target $S^{2}$. Geometrically, $\vec{B}$ is tangent to the closed curves $\chi=$ const (see e.g. [2, 3], 6, 17]; the authors of [17] describe Hopf curvatures slightly differently, by the Abelian projection of an $S U(2)$ pure gauge connection, which has some technical advantages). The Hopf index $N$ of $\chi$ may be computed from $\vec{B}$ via

$$
N=\frac{1}{16 \pi^{2}} \int d^{3} x \vec{A} \vec{B}
$$

where $\vec{B}=\vec{\partial} \times \vec{A}$.

The simplest (standard) Hopf map $\chi$ with Hopf index $N=1$ is

$$
\chi=\frac{2\left(x_{1}+i x_{2}\right)}{2 x_{3}-i\left(1-r^{2}\right)}
$$

with modulus and phase

$$
S^{2}=\frac{4\left(r^{2}-x_{3}^{2}\right)}{4 x_{3}^{2}+\left(1-r^{2}\right)^{2}}, \quad \sigma=\operatorname{atan} \frac{x_{2}}{x_{1}}+\operatorname{atan} \frac{1-r^{2}}{2 x_{3}}
$$

(a Hopf map has to be single valued, but may well be singular, as $\chi=\infty$ is just the south pole of the target $S^{2}$ ). This $\chi$, (15), leads to the Hopf curvature

$$
\vec{B}=\frac{16}{\left(1+r^{2}\right)^{2}} \vec{N}
$$

and we have introduced the unit vector

$$
\vec{N}=\frac{1}{1+r^{2}}\left(\begin{array}{c}
2 x_{1} x_{3}-2 x_{2} \\
2 x_{2} x_{3}+2 x_{1} \\
1-x_{1}^{2}-x_{2}^{2}+x_{3}^{2}
\end{array}\right)
$$

$\left(\vec{N}^{2}=1\right)$ for later convenience. 


\section{Construction of the Hopf instantons}

We start with the action $(i, j, k=1 \ldots 3)$

$$
S=\int d^{3} x\left(\Psi^{\dagger}\left(-i \partial_{j}-\bar{A}_{j}\right) \sigma_{j} \Psi+\frac{1}{2} \vec{A} \vec{B}\right)
$$

where $\Psi$ is a two-component spinor (Fermion), $\sigma_{j}$ are the Pauli matrices and $\vec{A}$ is an Abelian gauge potential. Further,

$$
S_{\mathrm{CS}}=\frac{1}{2} \int d^{3} x \vec{A} \vec{B}=\frac{1}{4} \int d^{3} x \epsilon_{i j k} A_{i} F_{j k}
$$

is the Chern-Simons (CS) action, where the Chern-Simons coupling constant is chosen equal to one; and

$$
\bar{A}_{i}=A_{i}+A_{i}^{\mathrm{B}}
$$

where the background gauge field $A_{i}^{\mathrm{B}}$ and its magnetic field $B_{i}^{\mathrm{B}}=\epsilon_{i j k} \partial_{j} A_{k}^{\mathrm{B}}$ are

$$
\vec{A}^{\mathrm{B}}=-\frac{1}{1+r^{2}} \vec{N}, \quad \vec{B}^{\mathrm{B}}=-\frac{4}{\left(1+r^{2}\right)^{2}} \vec{N}
$$

and the unit vector $\vec{N}$ is given in (18).

Observe that the (fixed, non-dynamical) background field is coupled to the Fermion, but it is absent in the CS term. The equations of motion resulting from the action (19) are

$$
\left(-i \partial_{j}-\bar{A}_{j}\right) \sigma_{j} \Psi=0
$$

(the Dirac equation) and

$$
\vec{\Sigma}:=\Psi^{\dagger} \vec{\sigma} \Psi=\vec{B}
$$

Observe that for any pair $\left(\Psi, \bar{A}_{j}\right)$ that solves the Dirac equation $(23)$ the spin density $\vec{\Sigma}$ related to $\Psi$ has to obey

$$
\vec{\partial} \vec{\Sigma}=0
$$

therefore, equations (23) and (24) are consistent.

The simplest solution to this system is (see [18, 19])

$$
\begin{gathered}
\Psi=\frac{4}{\left(1+r^{2}\right)^{\frac{3}{2}}}(\mathbf{1}+i \vec{x} \vec{\sigma})\left(\begin{array}{l}
1 \\
0
\end{array}\right) \\
\vec{A}=\frac{4}{1+r^{2}} \vec{N} \\
\vec{\Sigma}=\vec{B}=\frac{16}{\left(1+r^{2}\right)^{2}} \vec{N}
\end{gathered}
$$

( $\vec{N}$ is given in (18)). Here the dynamical gauge field is proportional to the background field, therefore one could find a solution without background field by choosing either 
a different normalization of the fermion (26) or by choosing a Chern-Simons coupling constant in (20), (24) different from 1. However, this will not be true for the solutions below, for which the background field (22) is crucial.

Further, the magnetic field (and spin density) of this simplest solution, (28), is precisely equal to the Hopf curvature (17) of the simplest standard Hopf map, (15). Here the question arises whether there are more solutions to $(23),(24)$ that are characterised by Hopf maps, and we already know from [1] that this is indeed the case. Here we shall generalise these results, therefore we should provide some more Hopf maps that will give rise to more solutions to (23), (24). We will produce these Hopf maps by composing the standard Hopf map with some maps $S^{2} \rightarrow S^{2}$, i.e.,

$$
\chi_{R}: S^{3} \stackrel{\chi^{(1)}}{\rightarrow} S^{2} \stackrel{R}{\rightarrow} S^{2}
$$

where $R(z)$ is a general rational map (3). Such Hopf maps have Hopf index $N=w^{2}$, where $w$ is the winding number (i.e., the degree (4) of the rational map).

Next we need some facts about the Dirac equation (23). Suppose a spinor $\Psi$ is given that solves (23) for some $\bar{A}_{i}$, then this gauge field $\bar{A}_{i}$ may actually be expressed in terms of the zero mode $\Psi$ as 18

$$
\begin{gathered}
\bar{A}_{i}=\frac{1}{|\vec{\Sigma}|}\left(\frac{1}{2} \epsilon_{i j k} \partial_{j} \Sigma_{k}+\operatorname{Im} \Psi^{\dagger} \partial_{i} \Psi\right) \\
=\frac{1}{2} \epsilon_{i j k}\left(\partial_{j} \ln |\vec{\Sigma}|\right) \mathcal{N}_{k}+\frac{1}{2} \epsilon_{i j k} \partial_{j} \mathcal{N}_{k}+\operatorname{Im} \widehat{\Psi}^{\dagger} \partial_{i} \widehat{\Psi}
\end{gathered}
$$

where we have expressed $\bar{A}_{i}$ in terms of the general unit vector and unit spinor

$$
\overrightarrow{\mathcal{N}}=\frac{\vec{\Sigma}}{|\vec{\Sigma}|}, \quad \widehat{\Psi}=\frac{\Psi}{\left|\Psi^{\dagger} \Psi\right|^{1 / 2}} .
$$

Now we are able to construct the solutions to (23), (24) as follows. We define the spinor

$$
\Psi^{(M)}=e^{i \Lambda} e^{M / 2} \Psi
$$

where $\Psi$ at the r.h.s. of (32) is just the zero mode of the simplest solution, (26). Further, $M$ is a real function of the simplest standard Hopf map $\chi,(15)$, and its complex conjugate $\bar{\chi} . \Lambda$ is a pure gauge factor that has to be chosen accordingly (see below).

For the corresponding spin density $\Sigma^{(M)}$ it still holds that

$$
\Sigma_{i, i}^{(M)}=e^{M}\left(\left(M_{, \chi \chi} \chi_{, i}+M_{, \bar{\chi}} \bar{\chi}_{, i}\right) \Sigma_{, i}+\Sigma_{i, i}\right)=0
$$

where $\Sigma_{i}$ is the spin density (and magnetic field) in (28), or equivalently the Hopf curvature (17) of the standard Hopf map (15). Therefore, $\Psi^{(M)}$ is still a zero mode. The corresponding gauge field $\bar{A}_{i}^{(M)}$ that solves the Dirac equation together with $\Psi^{(M)}$ may be computed with the help of (30) to be

$$
\bar{A}_{i}^{(M)}=\bar{A}_{i}+\frac{1}{2} \epsilon_{i j k}\left(\partial_{j} M\right) N_{k}+\Lambda_{, i}
$$


where $\bar{A}_{i}$ is the gauge field (27) of the simplest solution plus the background gauge field (22), and $\vec{N}$ is the specific unit vector (18). The corresponding magnetic field $\bar{B}_{i}^{(M)}=$ $\epsilon_{i j k} \partial_{j} \bar{A}_{k}^{(M)}$ is

$$
\begin{aligned}
\bar{B}_{l}^{(M)}= & \bar{B}_{l}+\frac{1}{2}\left[M_{, \chi}\left(\chi_{, l k} N_{k}+\chi_{, l} N_{k, k}-\chi_{, k k} N_{l}-\chi_{, k} N_{l, k}\right)+\right. \\
& M_{, \bar{\chi}}\left(\bar{\chi}_{, l k} N_{k}+\bar{\chi}_{, l} N_{k, k}-\bar{\chi}_{, k k} N_{l}-\bar{\chi}_{, k} N_{l, k}\right) \\
- & \left.\left(M_{, \chi \chi} \chi_{, k} \chi_{, k}+M_{, \bar{\chi} \bar{\chi}} \bar{\chi}_{, k} \bar{\chi}_{, k}+2 M_{, \chi \bar{\chi}} \chi_{, k} \bar{\chi}_{, k}\right) N_{l}\right]
\end{aligned}
$$

where $\bar{B}_{l}$ is the magnetic field (28) plus the background magnetic field (22). After some tedious algebra we find that only the coefficient of $M_{, \chi \bar{\chi}}$ is nonzero, i.e.,

$$
\begin{gathered}
\chi_{, l k} N_{k}+\chi_{, l} N_{k, k}-\chi_{, k k} N_{l}-\chi_{, k} N_{l, k}=0 \\
\chi_{, k} \chi, k=0 \\
\chi_{, k} \bar{\chi}_{, k}=8 \frac{(1+\chi \bar{\chi})^{2}}{\left(1+r^{2}\right)^{2}}
\end{gathered}
$$

and, therefore

$$
\bar{B}_{l}^{(M)}=\bar{B}_{l}-8 \frac{(1+\chi \bar{\chi})^{2}}{\left(1+r^{2}\right)^{2}} M_{, \chi \bar{\chi}} N_{l} .
$$

Now we should insert this into the Chern-Simons equation (24) after subtracting the background magnetic field (22),

$$
B_{l}^{(M)}=\bar{B}_{l}^{(M)}-B_{l}^{\mathrm{B}}=\Sigma_{l}^{(M)} .
$$

We arrive at

$$
\frac{16}{\left(1+r^{2}\right)^{2}} N_{l}-\frac{8(1+\chi \bar{\chi})^{2}}{\left(1+r^{2}\right)^{2}} M_{, \chi \bar{\chi}} N_{l}=\frac{16 e^{M}}{\left(1+r^{2}\right)^{2}} N_{l}
$$

or

$$
M_{, \chi \bar{\chi}}=-2 \frac{e^{M}-1}{(1+\chi \bar{\chi})^{2}}
$$

which is just version (12) of the Liouville equation (for $\widetilde{\rho}=(1+z \bar{z})^{2} \rho$ ). However, equation (42) holds in target space (i.e., for "coordinates" $\chi, \bar{\chi})$. Solutions to $(42)$ are therefore

$$
M=\ln \left((1+\chi \bar{\chi})^{2} \frac{\left|R^{\prime}(\chi)\right|^{2}}{(1+R(\chi) \bar{R}(\chi))^{2}}\right)
$$

for arbitrary rational functions $R(\chi)$.

Here we still have to explain why the restriction to rational maps $R(\chi)$ is necessary, because in principle any holomorphic function $f(\chi)$ in (43) solves eq. (42). This question is related to the pure gauge factor $\Lambda$ in (32) and (34), which we have not yet determined, because it did not show up in eq. (42), which is gauge invariant. 
The point is that the gauge field $\bar{A}_{l}^{(M)}$, as defined in (34), is singular at the zeros of $\exp (M(\chi, \bar{\chi}))$. In other words, $\bar{A}_{l}^{(M)}$ is singular along closed curves that are pre-images $\chi(\vec{x})=z_{i}$ of the zeros of $\exp (M)$. The $M$-dependent part of $\bar{A}_{l}^{(M)}$ in (34) may be rewritten as

$$
\frac{1}{2} \epsilon_{l j k} M_{, j} N_{k}=\frac{i}{2}\left(M_{, \chi} \chi_{, l}-M_{, \bar{\chi}} \bar{\chi}_{, l}\right)
$$

as may be checked easily. Now assume that $M$ is given by (43) for some $R(\chi)=$ $P(\chi) / Q(\chi)$, then the zeros of $\exp (M)$ are the zeros of

$$
\left|R^{\prime}(\chi)\right|^{2}=\left|P Q^{\prime}-P^{\prime} Q\right|^{2}
$$

and, generically, there are $p+q-1$ zeros (if we assume $p>q$, which we may, see below, then there are precisely $p+q-1$ zeros). Each individual zero in (45) looks like (with possible multiplicity $n$ )

$$
\left(\left(\chi-z_{i}\right)\left(\bar{\chi}-\bar{z}_{i}\right)\right)^{n}=: \zeta^{n} \bar{\zeta}^{n}
$$

which implies for the above expression (44)

$$
\frac{i}{2}\left(M_{, \chi \chi} \chi_{, l}-M_{, \bar{\chi}} \bar{\chi}_{, l}\right) \sim \frac{i n}{2} \frac{\bar{\zeta} \chi_{, l}-\zeta \bar{\chi}_{, l}}{\zeta \bar{\zeta}}+\ldots
$$

where the remainder is regular at $\zeta=0$. The above singularity may be compensated by the pure gauge factor

$$
\Lambda=-n \operatorname{atan} \frac{i(\zeta-\bar{\zeta})}{\zeta+\bar{\zeta}}
$$

Indeed $\left(\zeta_{, l} \equiv \chi_{, l}\right)$,

$$
\Lambda_{, l}=-\frac{i n}{2} \frac{\bar{\zeta} \zeta_{, l}-\zeta \bar{\zeta}_{, l}}{\zeta \bar{\zeta}}
$$

precisely cancels the singular term (47). The spinor in (32) is multiplied by the gauge factor $\exp (i \Lambda)$. This factor is single-valued only if the order $n$ of the zero of $P Q^{\prime}-P^{\prime} Q$ is integer, because $\Lambda$ in (48) is a multiply-valued function. This implies that both $P$ and $Q$ are polynomials and, therefore, restricts all zeros and poles of $R(z)$ to integer orders. If we demand, in addition, that the Hopf index (and, consequently, the Chern-Simons action) is finite, then $R(z)$ is restricted to the rational maps, as stated above.

In fact, this is not yet the whole story about singularities in $\bar{A}_{l}^{(M)}$. The point is that the expression

$$
\frac{i}{2} \frac{\bar{\chi} \chi, l-\chi \bar{\chi}, l}{\chi \bar{\chi}}
$$

is singular in the limit $\chi \rightarrow \infty$ as well, as may be checked easily. Further, the derivatives of the gauge factors, (48), for all the zeros of (45) produce this expression (50) for $\chi \rightarrow \infty$, because $\lim _{\chi \rightarrow \infty} \zeta=\chi$. As there are $p+q-1$ zeros (including multiplicities), the sum of all $\Lambda_{, l}$ in (49) behaves in the limit $\chi \rightarrow \infty$ as

$$
\lim _{\chi \rightarrow \infty} \sum_{\text {zeros,poles }} \Lambda_{, l}=-(p+q-1) \frac{i}{2} \frac{\bar{\chi} \chi, l-\chi \bar{\chi}_{, l}}{\chi \bar{\chi}} .
$$


In addition, eq. (44) produces a term that behaves in the limit $|\chi| \rightarrow \infty$ as

$$
-(p-q-1) \frac{i}{2} \frac{\bar{\chi} \chi_{, l}-\chi \bar{\chi}, l}{\chi \bar{\chi}}
$$

as may be checked easily. Therefore, alltogether we have to compensate (in the limit $|\chi| \rightarrow \infty)$

$$
-2(p-1) \frac{i}{2} \frac{\bar{\chi} \chi_{, l}-\chi \bar{\chi}_{, l}}{\chi \bar{\chi}}
$$

by an additional gauge transformation, without introducing further singularities at $\chi=0$.

Fortunately this is possible for the following reason. If we were to compensate (53) by the full gauge function

$$
\Lambda=2(p-1) \operatorname{atan} \frac{i(\chi-\bar{\chi})}{\chi+\bar{\chi}}=-2(p-1) \sigma
$$

(where $\sigma$ is the phase of $\chi$ given in (16)), this would introduce a singularity at $\chi=0$. However, $\sigma$ is the sum of two terms $\sigma=\sigma^{(1)}+\sigma^{(2)}$,

$$
\sigma^{(1)}=\operatorname{atan} \frac{x_{2}}{x_{1}}, \quad \sigma^{(2)}=\operatorname{atan} \frac{1-r^{2}}{2 x_{3}}
$$

where $\sigma_{, l}^{(1)}$ is singular at $\chi=0$ and $\sigma_{, l}^{(2)}$ is singular at $\chi=\infty$. Therefore, we may cancel the singularity of (53) at $|\chi|=\infty$ without introducing further singularities by performing an additional gauge transformation using only $\sigma^{(2)}$,

$$
\Lambda=-2(p-1) \sigma^{(2)} .
$$

\section{Summary}

We have shown that, in the presence of the fixed prescribed background magnetic field (22), there is an infinite number of fully three-dimensional solutions to the system of equations (23) and (24). These solutions are given by the set of Hopf maps (29), where the standard Hopf map (15) is followed by an arbitrary rational map (3); i.e., these solutions are Hopf instantons.

Before closing, we want to briefly discuss some aspects of our solutions. Firstly, one might ask whether the background gauge field (22) (and the corresponding magnetic field), which is crucial for our solutions to exist, admits some further interpretation. Indeed, as was already explained in [1], this background field may either be related to spin $1 / 2$ solutions of the Dirac equation (23), or it may be interpreted as a spin connection term in the Dirac operator on a conformally flat three-manifold with torsion, rather than as a background gauge field. For details we refer to [1].

Secondly, we want to mention that it is possible to count the number of solutions (43) for a given Hopf index $N=w^{2}$. As a solution is characterised by a rational map 
(3), which is the ratio of two polynomials, the complex coefficients $P_{i}$ and $Q_{i}$ of the two polynomials $P(z)=\sum P_{i} z^{i}$ and $Q(z)=\sum Q_{i} z^{i}$ parametrise these solutions. However, not each distinct rational map $R(z)$ leads to a different solution $M$, (43). In fact, $M$ is invariant under the $S U(2)$ transformation

$$
R(z) \rightarrow \frac{a R(z)+b}{-\bar{b} R(z)+\bar{a}}, \quad|a|^{2}+|b|^{2}=1, \quad a, b \in \mathbf{C} .
$$

Geometrically, this transformation corresponds to an $S O(3)$ rotation of the target $S^{2}$. Therefore, we should parametrise independent solutions $M$ by the set of punctured rational maps that leave one point invariant. If we choose the invariant point to be the south pole, i.e., $R(z=\infty)=\infty$, then this implies for the degrees $p$ and $q$ of the polynomials $P$ and $Q$ and for the Hopf index $N$

$$
N=p^{2}>q^{2}
$$

With this restriction, a general rational map $R(z)$ with fixed $p$ has $4 p$ real parameters, therefore we find a solution space of dimension $4 p$ for Hopf index $N=p^{2}$ for our class of solutions (43).

It should be mentioned at this point that this classification of the space of solutions is completely analogous to the case of the self-dual Jackiw-Pi model. In this model a non-relativistic scalar field is included in an Abelian Chern-Simons theory, and twodimensional solitonic solutions are found to exist [12, 13, 14, 21]. These soliton solutions obey the Liouville equation (8), however, in coordinate space rather than in target space. They are classified in a way that is completely analogous to our discussion above [21, with the magnetic flux as the topological quantity (where the magnetic flux is equal to $4 \pi$ times the degree $p$ of the rational map).

In fact, there is another relation to the self-dual Jackiw-Pi model. When our equations of motion (23) and (24) are dimensionally reduced by assuming independence of $x_{3}$ and by setting $A_{3} \equiv 0$, then the resulting equations of motion are precisely the equations of motion of the self-dual Jackiw-Pi model that we just described. These matters will be discussed in more detail elsewhere.

Finally we want to point out that our findings in no way imply that we have already exhausted the space of solutions. All our solutions are based on the simplest Hopf map

$\chi,(15)$, where the pre-image of a point $\chi=$ const is the simplest possible knot (the unknot). It is perfectly possible that by starting from more complicated Hopf maps, e.g., with more complicated knots as pre-images, one may find more solutions. This question is subject to further investigation.

\section{Acknowledgments}

The authors thank M. Fry for helpful discussions. In addition, CA thanks R. Jackiw for useful conversations, and especially for pointing out the relation to the Jackiw-Pi model. CA was supported by a Forbairt Basic Research Grant during part of the work. BM 
gratefully acknowledges financial support from the Training and Mobility of Researchers scheme (TMR no. ERBFMBICT983476).

\section{References}

[1] C. Adam, B. Muratori, C. Nash, hep-th/9909189

[2] A. F. Ranada, J. Phys. A25 (1992) 1621

[3] L. Faddeev and A. Niemi, Nature 387 (1997) 58

[4] L. Faddeev and A. Niemi, hep-th/9705176

[5] J. Gladikowski and M. Hellmund, Phys. Rev. D56 (1997) 5194

[6] R. Battye and P. Sutcliffe, hep-th/9811077

[7] R. Battye and P. Sutcliffe, Phys. Rev. Lett. 81 (1998) 4798

[8] H. Aratyn, L. A. Ferreira and A. H. Zimerman, Phys. Rev. Lett. 83 (1999) 1723

[9] S. Deser, R. Jackiw and S. Templeton, Ann. Phys. 140 (1982) 372

[10] R. Jackiw, K. Lee and E. J. Weinberg, Phys. Rev. D42 (1990) 3488

[11] R. Wang, Comm. Math. Phys. 137 (1991) 587

[12] R. Jackiw and S.-Y. Pi, Prog. Theor. Phys. (Suppl.) 107 (1992) 1

[13] R. Jackiw and S.-Y. Pi, Phys. Rev. Lett. 64 (1990) 2969; 66 (1991) 2682; Phys. Rev. D42 (1990) 3500

[14] G. Dunne, hep-th/9902115

[15] S. C. Zhang, T. H. Hansson and S. Kivelson, Phys. Rev. Lett. 62 (1989) 82

[16] J. K. Jain, Phys. Rev. Lett. 63 (1989) 199

[17] R. Jackiw and S.-Y. Pi, hep-th/9911072

[18] M. Loss and H.-Z. Yau, Comm. Math. Phys. 104 (1986) 283

[19] C. Adam, B. Muratori and C. Nash, Phys. Rev. D60 (1999) 125001

[20] C. Houghton, N. Manton and P. Sutcliffe, Nucl. Phys. B510 (1998) 507

[21] P. A. Horvathy, hep-th/9903116 